\title{
An Observational Study on Clinical and Liver Profile in Dengue Cases at a Tertiary Care Hospital in South India
}

Dr. V. Durga Bhargavi ${ }^{1}$, Dr. P. Brahmaiah ${ }^{2 *}$

${ }^{1}$ Post Graduate, Department of Paediatrics, Guntur Medical College, Kanna Vari Thota, Guntur, Andhra Pradesh 522004, India
${ }^{2}$ Assistant Professor, Department of Paediatrics, Guntur Medical College, Kanna Vari Thota, Guntur, Andhra Pradesh 522004, India

Article History
Received: 17.12 .2021
Accepted: 25.01 .2022
Published: 29.01 .2022
Journal homepage:
https://www.easpublisher.com
Quick Response Code

Abstract: Introduction: Dengue fever, the most common arthropod-borne viral illness in humans, is caused by the dengue virus, which is one of the world's most dangerous tropical infectious diseases. Dengue fever epidemics have been reported in India on various occasions. Liver involvement is known to occur in children with dengue fever. Liver dysfunction ranges from mild injury with elevated transaminase activity to severe injury with liver cell failure. Liver dysfunction varies in severity depending on the type of clinical presentation of dengue fever, but it is more common in children with severe dengue fever. Hence the following study was conducted to find out the range of liver dysfunction in children with dengue fever. Material and Methods: The study was conducted in a tertiary care hospital over a period of one year in a total of 100 patients who are serologically positive for dengue fever. Results: Patients were classified as dengue fever (DF)-48\%, and dengue hemorrhagic fever (DHF)-52\%. Liver function tests AST, ALT and ALP, bilirubin and albumin were deranged in children with dengue hemorrhagic fever. The mean total bilirubin, serum albumin, AST, ALT, ALP, PT, aPTT, INR, BT, CTwere $1.7 \mathrm{mg} / \mathrm{dl}, 2.9 \mathrm{mg} / \mathrm{dl}, 382 \mathrm{U} / \mathrm{L}, 240 \mathrm{U} / \mathrm{L}, 254.4 \mathrm{U} / \mathrm{L}, 39.8 \mathrm{~s}, 56.8 \mathrm{~s}, 1.5,4.6$ minutes and 2.5 minutes respectively. Derangement of liver function tests was significant among DHF as compared to DF. Conclusion: Liver dysfunction was very common in all forms of dengue fever, significantly deranged among DHF. AST levels raising significantly when compared to ALT.

Keywords: Dengue fever, Dengue hemorrhagic fever, Aspartate transaminase, Alanine Transaminase.

Copyright (C) 2022 The Author(s): This is an open-access article distributed under the terms of the Creative Commons Attribution 4.0 International License (CC BY-NC 4.0) which permits unrestricted use, distribution, and reproduction in any medium for non-commercial use provided the original author and source are credited.

\section{INTRODUCTION}

Dengue fever is still a major clinical and public health concern around the world. Dengue fever affects more than 2.5 billion people in the Tropics and Subtropics, each year an estimated 390 million cases were reported in roughly 125 countries [1,2]. During the monsoon and post-monsoon seasons, the incidence of dengue epidemics increases. The demographics most affected are children and young adults. It's a mosquitoborne Arboviral disease spread by the Aedes mosquito.

Thrombocytopenia is one of the most prevalent test results in Dengue [3]. Apart from thrombocytopenia, hepatic dysfunction is known to occur in Dengue, manifesting either as pain in right hypochondrium, hepatomegaly, jaundice or elevated aminotransferases [4-6]. It can cause liver dysfunction as a result of a direct viral action on liver cells and
Kupffer cells or as a result of a dysregulated host immunological response to the virus.

Pathophysiological changes involved here are viral cytopathy, hypoxic mitochondrial malfunction, immunological response, NV-induced TRAIL34, TNF, Fas signalling and increased endoplasmic reticular tension, finally leading hepatocyte to apoptotic phase. Severe types of Dengue fever had higher median Aspartate transaminase (AST) and also Alanine transaminase (ALT) values than uncomplicated Dengue fever. This could point to a link between higher transaminase levels and worsening disease severity. In contrast to the convalescent phase, the values of liver enzymes were shown to be higher in the febrile and severe phases of Dengue.

WHO (2009) Classification Dengue illness [7 ]:

- Undifferentiated fever

*Corresponding Author: Dr. P. Brahmaiah

Assistant Professor, Department of Paediatrics, Guntur Medical College, Kanna Vari Thota, Guntur, Andhra Pradesh 522004, India 
- $\quad$ Dengue fever (DF)

- Dengue hemorrhagic fever (DHF)

- Subcategorized into 4 severity grades

- Grades III and IV are defined as Dengue shock syndrome (DSS)

Since liver involvement is so common in paediatric dengue cases this study has been conducted in a tertiary care hospital to know the severity of liver involvement by measuring Transaminases in Dengue fever and Dengue haemorrhagic fever.

\section{Materials AND Method}

The hospital based study prospective observational study was conducted in a hundred patients with dengue fever to determine the profile of liver involvement in children with IgM positive Dengue viral infection.

\section{Inclusion Criteria}

1. Patients with serologically confirmed IgM positive Dengue viral fever.

2. Children between 1 to 12 years of age regardless of their gender.

\section{Exclusion Criteria}

1. Dengue-like diseases that are IgM negative

2. Children under the age of one year and above the age of twelve.

3. Children having a history of liver disease.

\section{Data Collection}

A proforma containing the sociodemographic data, history, symptoms was collected. The following investigations were done on the participants.

Complete blood counts, serum Dengue IgM , liver function tests such as serum bilirubin, AST, ALT, Alkaline phosphatase, total protein, serum albumin, prothrombin time and activated partial thromboplastin time were performed on all children enrolled in the study.

Informed verbal consent of the participants was taken. Ethical approval for conducting the study was obtained from Institutional Ethical Committee. Analysis was performed using the SPSS 12.0 software (SPSSInc., Chicago, IL USA)

All data are expressed as the mean $\pm \mathrm{SD}$ or as frequencies and proportions. Differences in the demographic and clinical data between patients with DF and DHF were tested by the chi-square test. Differences in laboratory data between patients with DF and DHF were analyzed using the Student's t-test for continuous variables. A P- value less than 0.05 was considered significant.

\section{RESULTS}

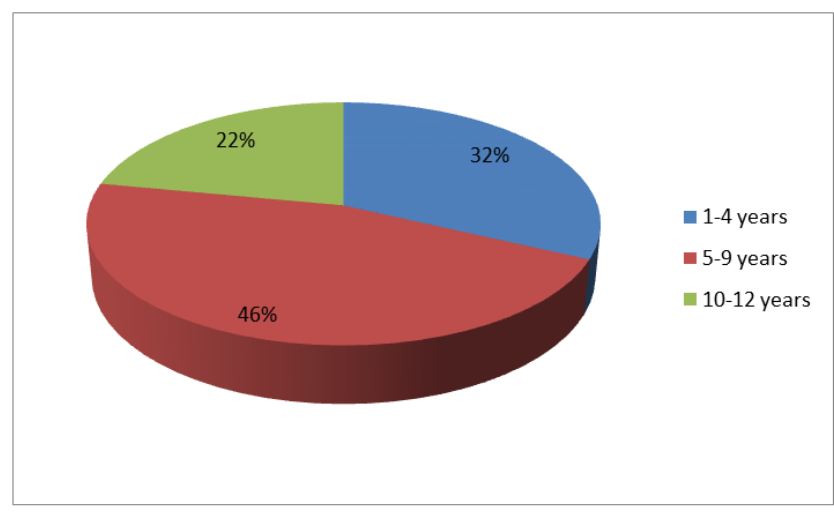

Fig 1: Age wise distribution of participants

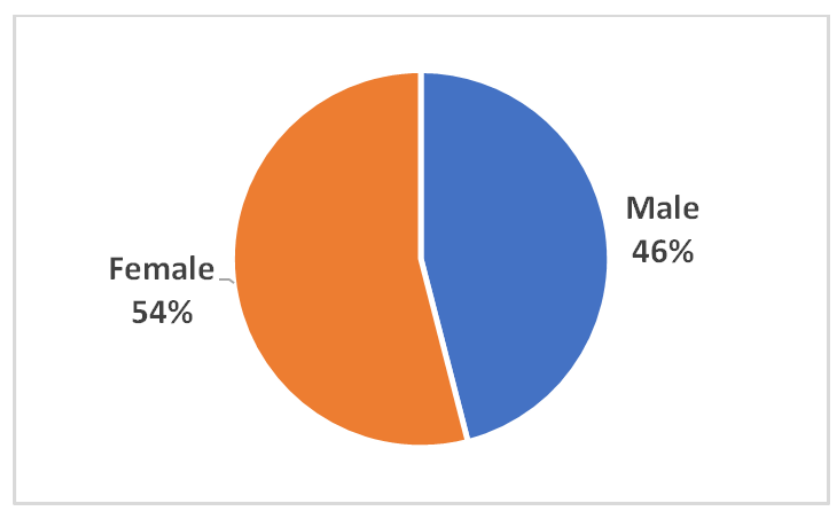

Fig 2: Gender distribution 


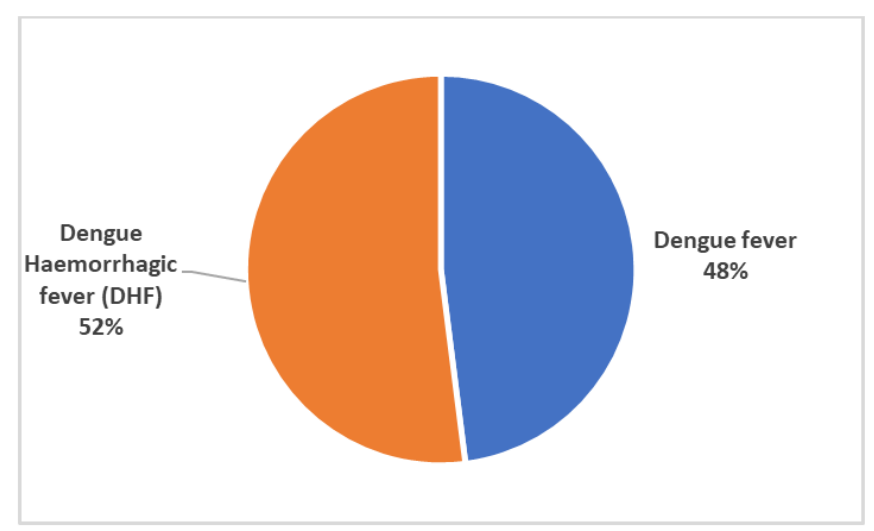

Fig 3: Distribution based on Diagnosis

In the present study out of 100 participants majority belong to $5-9 \mathrm{yr}$ age group. Only $22 \%$ of the study population belong to $10-12 \mathrm{yr}$ age group (Fig 1). The mean age of the study population was $6.52 \pm 3.51$. In this study, near-equal distribution was observed among the study population in relation to gender. $54 \%$ were female and $46 \%$ were males (Fig 2). Based on diagnosis, $52 \%$ were diagnosed as Dengue Haemorrhagic fever and $48 \%$ were diagnosed as Dengue fever (Fig 3). In this study, the mean age for the DF group was $6.16 \pm 4.03$ and the mean age for the DHF group was $7.32 \pm 3.80$.

Table 1: Demographic and clinical profile of DF and DHF groups

\begin{tabular}{|l|l|l|l|}
\hline & \multicolumn{2}{|l|}{ Diagnosis } & \multirow{2}{*}{ P value } \\
\hline & DF (n=48) & DHF (n=52) & \\
\hline Age Mean \pm SD & $6.16 \pm 4.03$ & $7.09 \pm 3.44$ & 0.06 \\
\hline Fever & $48(100 \%)$ & $52(100 \%)$ & 0.64 \\
\hline Headache & $21(43.75 \%)$ & $35(67.31 \%)$ & $0.01^{*}$ \\
\hline Vomiting & $24(50 \%)$ & $42(80.77 \%)$ & $0.01^{*}$ \\
\hline Pain abdomen & $26(54.17 \%)$ & $44(84.62 \%)$ & $0.001^{*}$ \\
\hline Arthralgia & $8(16.67 \%)$ & $15(28.85 \%)$ & 0.15 \\
\hline Malena & $2(4.17 \%)$ & $15(28.85 \%)$ & $0.001^{*}$ \\
\hline Rash & $41(85.42 \%)$ & $43(82.69 \%)$ & 0.71 \\
\hline Lymphadenopathy & $41(85.42 \%)$ & $43(82.69 \%)$ & 0.15 \\
\hline Edema & $12(25 \%)$ & $20(38.46 \%)$ & 0.15 \\
\hline Petechiae & $25(52.08 \%)$ & $39(75 \%)$ & $0.01^{*}$ \\
\hline Hepatomegaly & $46(95.83 \%)$ & $48(92.31 \%)$ & 0.46 \\
\hline Spleenomegaly & $18(37.5 \%)$ & $29(55.7 \%)$ & $0.04^{*}$ \\
\hline \multicolumn{4}{|l|}{ Chi-square test p<0.05 } \\
\end{tabular}

There was no statistically significant difference observed between the Diagnosis and Age distribution as the p-value was calculated to be $>0.05$. In this study, all the patients presented with fever, Headache was observed more among the DHF group (67.31\%) compared to the DF group (43.75\%). This finding was statistically significant as the p-value was calculated to be $<0.05$.

Vomiting was observed more among the DHF group $(80.77 \%)$ in contrast to DF group $(50 \%)$. This finding was statistically significant as the p-value was calculated to be $<0.05$.Pain abdomen was observed more among the DHF group $(84.62 \%)$ in contrast to DF group $(54.17 \%)$. This finding was statistically significant as the p-value was calculated to be $<0.05$.

Malena was observed more among the DHF group $(28.85 \%)$ in contrast to DF group $(4.17 \%)$. This finding was statistically significant as the p-value was calculated to be $<0.05$. In this study, Based on signs and their comparison between the study groups, petechiae and splenomegaly were observed to be statistically significant as the p-value was calculated to be $<0.05$ (Table 1). No statistically significant finding was observed between the symptoms of Arthralgia, Rash, between the study groups as the p-value was calculated to be $<0.05$. 
Table 2: Biochemical parameters between the study groups

\begin{tabular}{|c|c|c|c|}
\hline & \multicolumn{2}{|l|}{ Diagnosis } & \multirow[t]{2}{*}{$P$ value } \\
\hline & DF $(n=48)$ & DHF $(n=52)$ & \\
\hline $\mathrm{Hb}$ & $10.40 \pm 2.30$ & $10.11 \pm 2.25$ & $\begin{array}{l}\mathrm{t} \text { value }=0.63 \\
\mathrm{P}=0.52\end{array}$ \\
\hline HCT & $32.10 \pm 6.50$ & $29.56 \pm 6.86$ & $\begin{array}{l}\mathrm{t} \text { value }=1.89 \\
\mathrm{P}=0.06\end{array}$ \\
\hline Platelets & $1.76 \pm 0.83$ & $1.46 \pm 0.77$ & $\begin{array}{l}\mathrm{t} \text { value }=1.89 \\
\mathrm{P}=0.06\end{array}$ \\
\hline Total Bilirubin & $0.79 \pm 0.67$ & $2.45 \pm 0.15$ & $\begin{array}{l}\mathrm{t} \text { value }=17.40 \\
\mathrm{P}=0.0001 *\end{array}$ \\
\hline Direct Bilirubin & $0.27 \pm 0.58$ & $1.47 \pm 1.63$ & $\begin{array}{l}\mathrm{t} \text { value }=4.82 \\
\mathrm{P}=0.0001 *\end{array}$ \\
\hline Indirect Bilirubin & $0.51 \pm 0.13$ & $0.99 \pm 0.74$ & $\begin{array}{l}\mathrm{t} \text { value }=4.42 \\
\mathrm{P}=0.0001 *\end{array}$ \\
\hline S. Proteins & $6.30 \pm 0.83$ & $5.82 \pm 0.02$ & $\begin{array}{l}\mathrm{t} \text { value }=4.17 \\
\mathrm{P}=0.0001 *\end{array}$ \\
\hline Albumin & $3.17 \pm 0.52$ & $2.70 \pm 0.51$ & $\begin{array}{l}\mathrm{t} \text { value }=4.56 \\
\mathrm{P}=0.0001 *\end{array}$ \\
\hline AST & $117.63 \pm 124.56$ & $626.04 \pm 124.50$ & $\begin{array}{l}\mathrm{t} \text { value }=20.39 \\
\mathrm{P}=0.0001 *\end{array}$ \\
\hline ALT & $76.40 \pm 61.38$ & $392.10 \pm 61.14$ & $\begin{array}{l}\mathrm{t} \text { value }=25.74 \\
\mathrm{P}=0.0001 *\end{array}$ \\
\hline Alkaline phosphatase & $181.52 \pm 95.81$ & $321.69 \pm 95.75$ & $\begin{array}{l}\mathrm{t} \text { value }=194.07 \\
\mathrm{P}=0.0001 *\end{array}$ \\
\hline $\mathrm{PT}$ & $16.64 \pm 7.91$ & $61.10 \pm 7.80$ & $\begin{array}{l}\mathrm{t} \text { value }=28.28 \\
\mathrm{P}=0.0001 *\end{array}$ \\
\hline APTT & $35.82 \pm 7.99$ & $76.12 \pm 7.96$ & $\begin{array}{l}\mathrm{t} \text { value }=25.24 \\
\mathrm{P}=0.0001 *\end{array}$ \\
\hline INR & $1.43 \pm 0.74$ & $1.64 \pm 0.48$ & $\begin{array}{l}\mathrm{t} \text { value }=1.69 \\
\mathrm{P}=0.09\end{array}$ \\
\hline
\end{tabular}

Student $\mathrm{t}$ test $\mathrm{p}$ value $<0.05$

In this study, a statistically significant difference was observed between the study groups in relation to Total Bilirubin, Direct Bilirubin, Indirect Bilirubin, serum proteins, Albumin, AST, ALT, Alkaline Phosphatase, PT, APTT, BT as the p-value calculated to be $<0.05$ (Table 2).

\section{DISCUSSION}

Dengue fever, also known as "Break-bone fever," has become one of the most common causes of feverish disease in tropical and subtropical areas. Dengue fever is a mosquito-borne disease that is second only to malaria as one of the most common cause of Arboviral disease worldwide.

\section{Age}

In the study conducted by Jagadish Kumar K et al., [8] the majority of the participants were aged around 5 years and by Carlos et al., [9] the participants mean age was around $9.8 \pm 4.0$ years and by Dussart $P$ et al., [10] the participants mean age was $8.2 \pm 3.3$ years, these findings were in consonance with this study.

\section{Gender}

In the study conducted by Carlos et al., [9] the male-female ratio was 1.49 and this was in close consonance with the present study.

\section{Clinical Profile}

In the study conducted by Carlos et al., [9] the majority were cases of Dengue fever and $33.4 \%$ were cases of DHF and this distribution was in close consonance with the present study.

In the study conducted by Carlos et al., [9] A significant increase in the frequency of abdominal pain was found among the DHF group before admission $(P<$ 0.008 ) and at the time of admission compared with the DF group

\section{Transaminases}

Praveen M et al., [11] reported that $100 \%$ of patients out of 60 participants had elevated AST and $85 \%$ patients had elevated ALT. The albumin level was low in $75 \%$ patients. Average AST was 316 units/L and average ALT was 156 units/L.

Mohan et al., reported that AST and ALT levels were raised during the initial 1 st and 2 nd week, 
respectively. In compared to conventional Dengue fever, hepatic impairment in the form of markedly increased enzymes was found to be more common in severe and complicated Dengue [12].

According to Kuo et al., it was $93.3 \%$ [13] but as per Nguyen et al., it was $97.7 \%$ [14] of Dengue infection cases had increased AST levels.

In a study by Sedhain et al., liver enzyme SGOT was increased with the value of more than 50 $\mathrm{IU} / \mathrm{mL}$ in $78.4 \%$ and $96.5 \%$ of cases of DF and DHF, respectively [15].

In a study by Gandhi et al., (85\%) of the patients had an elevation of at least one of the liver enzymes $(P<0.001)$ with alterations in SGOT [16].

According to Roy et al., there was 84.4 percent and 93.75 percent ALT and AST elevation in DF with a warning sign, and 94.5 percent and 95.9 percent ALT and AST elevation in severe Dengue and acute hepatic failure in the severe Dengue group [17].

In a major study from Brazil, elevated AST and ALT levels were found in 63.4 percent and 45 percent of patients, respectively, out of 1585 Dengue cases, with 3.8 percent of cases having a 10 fold higher in transaminase level. Other authors have reported a similar increase in liver enzymes of more than 10 fold in adults, ranging from $1.8 \%$ to $11.2 \%$ respectively, had elevated AST and ALT levels [18].

Petdachai et al., reported that levels of AST were higher than those of ALT [19].

In our study 44 percent of DSS, 22.8 percent of DHF, and just 3.4 percent of the DF group, we detected a higher than 10-fold increase in AST. This study found a more than 10-fold increase in ALT in 16 percent of DSS, 7.7\% of DHF, and $0 \%$ of DF participants. Transaminases levels were found to be more than 10 times higher in the DSS and DHF groups than in the DF group, which was statistically important.

In the current investigation, most of our patients exhibited elevated liver enzymes with SGOT values being higher than SGPT values, as in previous studies. Hepatic enzyme impairment was more common in patients with severe Dengue.

\section{CONCLUSION}

Dengue is one of the most common viral illness in India, significantly affecting paediatric population. Among children liver is one of the common organ that is affected ranging from elevated Transaminases to Liver cell failure depending on severity of Dengue illness.
Among those with Dengue haemorrhagic fever Transaminases especially AST when compared to ALT and ALP is one which is significantly elevated when compared to Dengue fever.

Elevated serum bilirubin and coagulation profile (PT, aPTT, INR) with increased incidence and severity of vomitings, abdominal pain, spleenomegaly, petechiae and malena are significantly associated with DHF than DF.

Elevation of Transaminases specifically AST and these clinical symptoms can be taken as early predictors for progression into DHF in order to prevent mortality in paediatric population by early commencement of appropriate supportive care especially in those with liver involvement $[20,21]$.

\section{REFERENCES}

1. Nishino, K., Luce, R., Mendez, J. A., Garnier, S., Milot, V., \& Garcia, E. Dengue vaccine: WHO position paper-July 2016 Weekly Epidemiological Record 2016; 91(30): 349-64.

2. Bhatt, S., Gething, P. W., Brady, O. J., Messina, J. P., Farlow, A. W., Moyes, C. L., ... \& Hay, S. I. (2013). The global distribution and burden of dengue. Nature, 496(7446), 504-507.

3. Simmons, C. P., Farrar, J. J., van Vinh Chau, N., \& Wills, B. (2012). Dengue. New England Journal of Medicine, 366(15), 1423-1432.

4. Chhina, R. S., Goyal, O., Chhina, D. K., Goyal, P., Kumar, R., \& Puri, S. (2008). Liver function tests in patients with dengue viral infection. Dengue Bulletin, 32, 110-117.

5. Itha, S., Kashyap, R., Krishnani, N., Saraswat, V. A., Choudhuri, G., \& Aggarwal, R. (2005). Profile of liver involvement in dengue virus infection. National Medical Journal of India, 18(3), 127-130.

6. Parkash, O., Almas, A., Jafri, S. M., Hamid, S., Akhtar, J., \& Alishah, H. (2010). Severity of acute hepatitis and its outcome in patients with dengue fever in a tertiary care hospital Karachi, Pakistan (South Asia). BMC gastroenterology, 10(1), 1-8.

7. World Health Organization. (1978). Special program for research and training in tropical diseases.

8. Jagadishkumar, K., Jain, P., Manjunath, V. G., \& Umesh, L. (2012). Hepatic involvement in dengue fever in children. Iranian journal of pediatrics, 22(2), 231.

9. Carlos, C. C., Oishi, K., Cinco, M. T., Mapua, C. A., Inoue, S., Cruz, D. J. M., ... \& Nagatake, T. (2005). Comparison of clinical features and hematologic abnormalities between dengue fever and dengue hemorrhagic fever among children in the Philippines. The American journal of tropical medicine and hygiene, 73(2), 435-440.

10. Chongsrisawat, V., Hutagalung, Y., \& Poovorawan, Y. (2009). Liver function test results 
and outcomes in children with acute liver failure due to dengue infection. Southeast Asian journal of tropical medicine and public health, 40(1), 47.

11. Praveen, M., \& Girish, I. (2018). Hepatic Dysfunction in Dengue Fever-A Prospective Study Done At a Tertiary Care Hospital, Shimoga. Journal of Evolution of Medical and Dental Sciences-JEMDS, 7(1), 50-52.

12. Mohan, B., Patwari, A. K., \& Anand, V. K. (2000). Brief report. Hepatic dysfunction in childhood dengue infection. Journal of tropical pediatrics, 46(1), 40-43.

13. Kuo, C. H., Tai, D. I., Chang-Chien, C. S., Lan, C. K., Chiou, S. S., \& Liaw, Y. F. (1992). Liver biochemical tests and dengue fever. The American journal of tropical medicine and hygiene, 47(3), 265-270.

14. Nguyen, T. L., Nguyen, T. H., \& Tieu, N. T. (1997). The impact of dengue haemorrhagic fever on liver function. Research in virology, 148(4), 273-277.

15. Sedhain, A., Bhattarai, G. R., Adhikari, S., Shrestha, B., \& Sapkota, A. (2013). Liver involvement associated with dengue infection during a major outbreak in central Nepal. Journal of Advances in Internal Medicine, 2(2), 42-46.
16. Gandhi, K., \& Shetty, M. (2013). Profile of liver function test in patients with dengue infection in South India. Medical Journal of Dr. DY Patil University, 6(4), 370-372.

17. Roy, A., Sarkar, D., Chakraborty, S., Chaudhuri, J., Ghosh, P., \& Chakraborty, S. (2013). Profile of hepatic involvement by dengue virus in dengue infected children. North American journal of medical sciences, 5(8), 480-485.

18. Kuo, C. H., Tai, D. I., Chang-Chien, C. S., Lan, C. K., Chiou, S. S., \& Liaw, Y. F. (1992). Liver biochemical tests and dengue fever. The American journal of tropical medicine and hygiene,47(3), 265-270.

19. Petdachai, W. (2005). Hepatic Dysfunction in Children with Dengue Shock Syndrome. Dengue Bulletin, 29, 112-127.

20. Wong, M., \& Shen, E. (2008). The utility of liver function tests in dengue. Annals of the Academy of Medicine, Singapore, 37(1), 82-83.

21. Kamath, S. R., \& Ranjit, S. (2006). Clinical features, complications and atypical manifestations of children with severe forms of dengue hemorrhagic fever in South India. The indian journal of pediatrics, 73(10), 889-895.

Cite This Article: V. Durga Bhargavi \& P. Brahmaiah (2022). An Observational Study on Clinical and Liver Profile in Dengue Cases at a Tertiary Care Hospital in South India. East African Scholars J Med Sci, 5(1), 23-28. 